\title{
Noninvasive Assessment of Fibrosis in Patients with Nonalcoholic Fatty Liver Disease
}

\author{
Elena Buzzetti, Rosa Lombardi, Laura De Luca, and Emmanuel A. Tsochatzis
}

UCL Institute for Liver and Digestive Health, Royal Free Hospital and UCL, London NW3 2QG, UK

Correspondence should be addressed to Emmanuel A. Tsochatzis; e.tsochatzis@ucl.ac.uk

Received 22 January 2015; Accepted 17 April 2015

Academic Editor: Dario Iafusco

Copyright (c) 2015 Elena Buzzetti et al. This is an open access article distributed under the Creative Commons Attribution License, which permits unrestricted use, distribution, and reproduction in any medium, provided the original work is properly cited.

\begin{abstract}
Nonalcoholic fatty liver disease (NAFLD) is prevalent in $20-25 \%$ of the general population and is associated with metabolic risk factors such as obesity, diabetes mellitus, and dyslipidemia. Histologically, NAFLD ranges from simple steatosis to nonalcoholic steatohepatitis (NASH), fibrosis, and cirrhosis. As NASH develops in only 10-15\% of patients with NAFLD, it is not practical to biopsy all patients who present with NAFLD. Noninvasive fibrosis tests have been extensively developed recently and offer alternatives for staging fibrosis. Despite their increasing use, such tests cannot adequately differentiate simple steatosis from NASH. At present, such tests can be used as first line tests to rule out patients without advanced fibrosis and thus prevent unnecessary secondary care referrals in a significant number of patients. In this review we present the evidence for the use of noninvasive fibrosis tests in patients with NAFLD.
\end{abstract}

\section{Introduction}

Nonalcoholic fatty liver disease (NAFLD) is the most common cause of abnormal liver function tests in primary care in industrialized countries. It is associated with insulin resistance (IR) and is considered as the hepatic manifestation of the metabolic syndrome (MeS) [1].

The reported prevalence of NAFLD varies widely depending on the studied population, the diagnostic modality, and the definition [2]. According to the latest data, $20-30 \%$ of European and Middle East population have NAFLD, with an incidence of 39/100.000 inhabitants in the UK [3]. In USA the prevalence of NAFLD as assessed by MR spectroscopy is 34\%, while it is lower in the Far East (15\%) [4].

Ultrasonography, in association with routine laboratory investigations, is an acceptable first line test to identify patients with NAFLD, even if it has suboptimal sensitivity and reproducibility, being an operator-dependent technique [4].

From a histological point of view, NAFLD encompasses a wide spectrum of liver pathology, from simple steatosis to steatohepatitis, fibrosis, and cirrhosis [5]. While simple steatosis is associated with a low risk of progression, patients with NASH, estimated to be $15-20 \%$ of the NAFLD population, have increased risk of liver-related morbidity and mortality from cirrhosis and development of HCC $[6,7]$. Other metabolic risk factors, such as the presence of metabolic syndrome, are associated with more severe liver disease [8].

The pivotal issue in the management of patients with NAFLD is the diagnosis of steatohepatitis and fibrosis at an early stage. This would allow the identification of those patients who require stricter follow-up, targeted lifestyle interventions for weight reduction, management of the components of the metabolic syndrome, and eventual inclusion in trials of new treatment strategies [9].

Liver biopsy is the gold standard for diagnosing NASH and assessing fibrosis, though its limitations are well known: potential risk of sampling errors, intra- and interobserver variability, invasiveness, and scarce tolerability by the patient $[10,11]$. These characteristics, together with the high prevalence of hepatic steatosis and its low risk of progression in the majority of affected people, make liver biopsy an inappropriate first line tool for diagnosis in unselected patients.

Noninvasive liver fibrosis tests have been extensively developed recently and offer alternatives for staging fibrosis. 
TABLE 1: Noninvasive fibrosis serum tests and scores to diagnose nonalcoholic steatohepatitis (NASH) and/or stage fibrosis in patients with nonalcoholic fatty liver disease (NAFLD) [12].

\begin{tabular}{|c|c|c|c|c|}
\hline Test & Variables & NAFLD stage assessed & Cut-off & AUROC \\
\hline AST: ALT ratio & ALT and AST serum levels & $\mathrm{F} 4$ & 1 & NA \\
\hline CK-18 & Cytokeratin 18 fragments & $\begin{array}{c}\text { NAFLD } \\
\text { NASH } \\
\text { Fibrosis (any degree) }\end{array}$ & NA & $\begin{array}{c}0.77 \\
0.65-0.83 \\
0.68\end{array}$ \\
\hline Ferritin & Serum ferritin & $\begin{array}{c}\mathrm{NASH} / \geq \mathrm{F} 2 \\
\geq \mathrm{F} 3\end{array}$ & $\begin{array}{l}1.5 \mathrm{ULN} \\
2.5 \mathrm{ULN}\end{array}$ & $\begin{array}{l}0.57 \\
\text { NA }\end{array}$ \\
\hline $\begin{array}{l}\text { NASH } \\
\text { diagnostic }\end{array}$ & CK-18 fragments, adiponectin, and resistin & NASH & 0.43 & $0.70-0.85$ \\
\hline $\begin{array}{l}\text { NASH } \\
\text { diagnostic panel }\end{array}$ & $\begin{array}{l}\text { Gender, BMI, diabetes, triglycerides, and } \\
\text { CK-18 (total levels and fragments) }\end{array}$ & NASH & NA & 0.81 \\
\hline NashTest & $\begin{array}{l}\text { Age, gender, weight, height, bilirubin, GGT, } \\
\alpha 2 \text {-macroglobulin, apolipoprotein A1, } \\
\text { haptoglobin, AST, serum glucose, } \\
\text { triglycerides, and cholesterol }\end{array}$ & $\begin{array}{l}\text { No NASH } \\
\text { Borderline NASH } \\
\text { NASH }\end{array}$ & NA & $\begin{array}{l}\text { NA } \\
\text { NA } \\
0.69-0.83\end{array}$ \\
\hline $\begin{array}{l}\text { NAFIC scoring } \\
\text { system }\end{array}$ & $\begin{array}{l}\text { Ferritin } \geq 200 \text { or } \geq 300 \mathrm{ng} / \mathrm{mL}(\mathrm{F} \text { or } \mathrm{M})=1 \\
\text { Serum fasting insulin } \geq 10 \mu \mathrm{U} / \mathrm{mL}=1 \\
\text { Serum type IV collagen } 7 \mathrm{~s} \geq 5.0 \mathrm{ng} / \mathrm{mL}=2\end{array}$ & $\mathrm{NASH}$ & 2 & $0.78-0.85$ \\
\hline $\begin{array}{l}\text { Modified } \\
\text { NAFIC } \\
\text { scoring system }\end{array}$ & $\begin{array}{l}\text { Ferritin } \geq 200 \text { or } \geq 300 \mathrm{ng} / \mathrm{mL}(\mathrm{F} \text { or } \mathrm{M})=1 \\
\text { Serum fasting insulin } \geq 10<15 \mu \mathrm{U} / \mathrm{mL}=1 \\
\text { Serum fasting insulin } \geq 15=2 \\
\text { Serum type IV collagen } 7 \mathrm{~s} \geq 5.0 \mathrm{ng} / \mathrm{mL}=2\end{array}$ & NASH & 2 & 0.80 \\
\hline PIIINP & Terminal peptide of procollagen III levels & $\begin{array}{l}\text { NASH } \\
\geq \mathrm{F} 3\end{array}$ & $\begin{array}{l}6.6 \mathrm{ng} / \mathrm{mL} \text { (low cut-off) } \\
11 \mathrm{ng} / \mathrm{mL} \text { (high cut-off) }\end{array}$ & $\begin{array}{l}0.82 \\
0.84\end{array}$ \\
\hline APRI & AST, PLT & $\geq \mathrm{F} 2$ & $\begin{array}{l}0.45 \text { (low cut-off) } \\
1.5 \text { (high cut-off) }\end{array}$ & $0.62-0.94$ \\
\hline BARD & BMI, AST/ALT ratio, and diabetes & $\geq \mathrm{F} 3$ & $\geq 2$ & 0.80 \\
\hline BARDI & BMI, AST/ALT ratio, diabetes, and INR & $\geq \mathrm{F} 3$ & $\geq 3$ & 0.88 \\
\hline $\begin{array}{l}\text { NAFLD fibrosis } \\
\text { score }\end{array}$ & $\begin{array}{l}\text { Age, BMI, diabetes. AST, ALT, platelet } \\
\text { count, and albumin }\end{array}$ & $\geq \mathrm{F} 3$ & $\begin{array}{l}-1.45 \text { (low cut-off) } \\
0.67 \text { (high cut-off) }\end{array}$ & $0.82-0.88$ \\
\hline FIB-4 & Age, AST, ALT, and platelet count & $\geq \mathrm{F} 3$ & $\begin{array}{l}1.3-1.92 \text { (low cut-off) } \\
3.25 \text { (high cut-off) }\end{array}$ & $0.87,0.88$ \\
\hline Hepascore & $\begin{array}{l}\text { Age, sex, bilirubin, GGT, } \alpha 2 \text {-macroglobulin, } \\
\text { and hyaluronic acid }\end{array}$ & $\begin{array}{l}\geq \mathrm{F} 2 \\
\geq \mathrm{F} 3 \\
\mathrm{~F} 4\end{array}$ & $\begin{array}{c}0.44 \\
0.37 \\
0.7\end{array}$ & $\begin{array}{c}0.73 \\
0.81 \\
0.9\end{array}$ \\
\hline ELF test & $\begin{array}{l}\text { Hyaluronic acid (HA), PIIINP, and tissue } \\
\text { inhibitor of metalloproteinase } 1 \text { (TIMP-1). }\end{array}$ & $\begin{array}{l}\geq \mathrm{F} 2 \\
\geq \mathrm{F} 3 \\
\mathrm{~F} 4\end{array}$ & $\begin{array}{c}8.5-10.18 \\
10.35(9.33-10.51) \\
9.35-11.3\end{array}$ & $\begin{array}{l}0.82 \\
0.90 \\
0.87\end{array}$ \\
\hline FibroTest & $\begin{array}{l}\text { Haptoglobin, } \alpha 2 \text {-macroglobulin, } \\
\text { apolipoprotein-A, bilirubin, and GGT }\end{array}$ & $\geq \mathrm{F} 3$ & $\begin{array}{l}0.3 \text { (low cut-off) } \\
0.7 \text { (high cut-off) }\end{array}$ & 0.88 \\
\hline FibroMeter & $\begin{array}{l}\text { Age, weight, glucose, AST, ALT, PLT, and } \\
\text { ferritin }\end{array}$ & $\begin{array}{c}\geq \mathrm{F} 2 \\
\geq \mathrm{F} 3 \\
\mathrm{~F} 4\end{array}$ & $\begin{array}{l}\text { F3: } 0.61 \text { (low cut-off) } \\
0.71 \text { (high cut-off) }\end{array}$ & $\begin{array}{c}0.94 \\
0.93 \\
0.9\end{array}$ \\
\hline
\end{tabular}

ALT: alanine aminotransferase; AST: aspartate aminotransferase; AUROC: area under receiver operator characteristic curve; CK-18: cytokeratin 18; NA: not available; BMI: body mass index; GGT: $\gamma$-glutamyl transpeptidase; PLT: platelets; PIIINP: terminal peptide of procollagen III; APRI: aspartate aminotransferase to platelets ratio; INR: international normalized ratio; ELF: enhanced liver fibrosis; ULN: upper limit of normal.

In this review, we discuss the evidence for their use in patients with NAFLD.

\section{Noninvasive Fibrosis Tests}

In the last years, we have witnessed explosive development and use of noninvasive fibrosis tests (Table 1). These can be divided into three categories, namely, simple or indirect serum markers, direct serum markers, and imaging modalities [13].

(i) Indirect or class II serum markers consist of routine biochemical tests such as transaminases, platelet count, and albumin that are usually combined with demographic characteristics potentially linked to liver fibrosis such as age or diabetes [14]. 
Indirect serum markers and panels usually have dual cutoffs: a high cut-off with high specificity and a low cut-off with high sensitivity. If these cut-offs are combined, depending on the clinical scenario and prevalence of the examined disease, the numbers of false positive and false negatives are minimized. However, a proportion of patients will fall in the indeterminate range of values and will therefore need further testing.

(ii) Direct or class I serum markers detect the fibrogenic process and the extracellular matrix turnover. Such markers include hyaluronic acid, collagenases and their inhibitors (TIMP), and profibrotic cytokines (TGF beta) [15]. Although they perform better than indirect serum markers, their sensitivity is low in the initial stages of fibrosis and they are not routinely available in most clinical laboratories [14].

(iii) Imaging modalities measure the elasticity and/or stiffness of the liver tissue with MR and US techniques.

Transient elastography (Fibroscan, Echosens, Paris, France) is a noninvasive US-based technique in which a lowfrequency $(50 \mathrm{~Hz})$ elastic shear wave is generated by a transducer and then propagates through the tissues. The speed of propagation is proportional to the stiffness of the crossed tissue and its measurement can, through specific software, provide values of liver stiffness expressed in kilopascal $(\mathrm{kPa})$ [16]. The final value is the median of 10 valid measurements.

TE is painless and rapid and thus highly acceptable to patients. The volume of liver studied with this method is a cylinder of $1 \times 4 \mathrm{~cm}$ length, therefore 100 times bigger than a sample taken by liver biopsy.

TE has already been incorporated in the international guidelines for $\mathrm{CHB}$ and $\mathrm{CHC}$ for pretreatment fibrosis assessment $[17,18]$ and it has also been evaluated as a potential technique to predict the presence and degree of portal hypertension $[19,20]$ and HCC [21].

In ARFI (acoustic radiation force impulse), the elastography system is integrated on an ultrasound machine, allowing the operator to select the site of liver stiffness measurement (LSM) through a real-time B-mode ultrasonography and localize the shear wave avoiding vessels and ribs.

This technique showed a similar accuracy when compared to Fibroscan [22-24] in detecting fibrosis and/or cirrhosis. The range of measured values is narrower than Fibroscan, while quality criteria for ARFI have not yet been established.

The supersonic shear imaging (SSI) or Shear Wave Elastography is also built on a US device but, while ARFI is based on the emission of a single pulse wave, in SSI the transducer emits several wave fronts at increasing depth, with a frequency band ranging from 60 to $6000 \mathrm{~Hz}$.

It generates real-time color mapping of the elasticity, which is integrated in a standard B-mode image, allowing a quantitative representation of the tissue elasticity. The final value will be the average of many obtained by selecting the region of measurement with guidance from both the B-mode and SWE images [25].

Magnetic resonance elastography (MRE) combines the ability of elastography to provide information about liver tissue texture to the advantages of MR, which allows the assessment of the entire liver rather than a small part [26].
The main limitation of all the above noninvasive liver fibrosis tests is the absence of uniformly established and validated cut-offs for different disease etiologies and fibrosis stages.

\section{Noninvasive Diagnosis of NASH}

3.1. Liver Enzymes. Both serum levels of aspartate aminotransferase (AST) and alanine aminotransferase (ALT) may increase in patients with NAFLD and this is more prominent for ALT rather than AST [27]. However, the increase is not proportionate to the liver inflammation or fibrosis and it is well documented that patients with significant fibrosis may present with normal transaminases [28].

Data from different studies report a low accuracy for ALT levels in predicting NASH, with an area under the curve (AUC) of $0.6[29,30]$. Reducing the upper limit of normal cut-off value for ALT from 30 to $19 \mathrm{U} / \mathrm{L}$ showed an improvement of sensitivity in a cohort of 233 patients undergoing bariatric surgery, but at the expense of specificity [31].

3.2. Cytokeratin 18. In the last ten years, emerging data suggested that hepatocellular apoptosis, a genetically determined form of cell death, may have a significant role in the transition from simple steatosis to NASH [32]. Within the apoptotic process, various caspases are activated with the subsequent cleavage of different substrates. The main substrate in the liver is cytokeratin 18 (CK-18), which is an intermediate filament protein.

In total there have been more than eight studies exploring the diagnostic accuracy of CK-18 fragments levels in NASH. The main issue is that the cut-offs for diagnosis of NASH varied among studies; therefore this remains an unvalidated test [6]. In a cohort of 139 patients with biopsy-proved NAFLD compared to 155 healthy controls, CK-18 fragments levels independently predicted the presence of NASH (AUC 0.83 ) with a sensitivity of 0.75 and specificity of 0.9 [33]. Similar results were obtained by the same group in a cohort of 201 pediatric patients [34].

In a recent study, involving a multiethnic population of 424 patients, CK-18 fragments levels showed a high specificity for NAFLD and fibrosis but a limited sensitivity/specificity for NASH (58\%/68\%) [35].

Therefore, CK-18 is not an adequate screening tool to discriminate NASH or early fibrosis in NAFLD patients.

3.3. PIIIPN. Terminal peptide of procollagen III (PIIIPN), involved in fibrogenic processes, was evaluated in a single validation study on 136 subjects with NAFLD (71 in validation cohort) as marker of NASH and developing fibrosis. Although its performance was promising, with an AUC of 0.85-0.87, further validation studies in larger cohorts are needed [36].

3.4. Panel Tests. NASH diagnostic is a proprietary test which encompasses serum levels of cleaved CK-18, adiponectin, and resistin. It was tested in a cohort of 101 patients that also included a validation group of 32 patients and showed a moderate reliability for discriminating NASH from simple 
steatosis (AUC of 0.85 and 0.73 in the derivation and validation cohort, resp.) [15].

The same panel was reevaluated in an independent sample of 79 patients and had a suboptimal AUC of 0.7. Thus, a new model was subsequently developed by the same group, namely, the NASH diagnostic panel that consists of presence of diabetes, gender, BMI, serum triglycerides, CK18 fragments, and total CK-18 levels. Although the AUC was greater $(0.8)$ than those for NASH diagnostics or CK-18 fragment levels alone (0.7 and 0.71, resp.) [37], this test needs further validation in independent cohorts.

The NashTest comprises 13 serum and clinical parameters, namely, total bilirubin, GGT, $\alpha 2$-macroglobulin, apolipoprotein Al, haptoglobin, age, gender, weight, height, AST, serum glucose, triglycerides, and cholesterol, and divides patients into three categories: not NASH, borderline NASH, and NASH [38]. NashTest showed a low sensitivity but high specificity in diagnosing NASH with an AUC ranging between 0.7 and 0.83 [38]. NashTest was also used in patients with hyperlipidaemia and morbid obesity and showed AUCs of 0.8 and 0.77 , respectively, for the diagnosis of NASH $[39,40]$. This test needs external validation in independent cohorts.

NAFIC score was derived and validated in a cohort of 117 and 442 Japanese biopsy-proven NAFLD patients [41]. The score, ranging from 0 to 4 , was calculated considering cut-off values for serum ferritin, serum fasting insulin, and serum type collagen $7 \mathrm{~s}$. The AUC for predicting NASH was 0.85 and 0.78 in the estimation group and validation group, respectively [41].

In a backward validation study published in 2013, Nakamura et al. showed that the sensitivity of the NAFIC score for diagnosing NASH could further improve by adding different insulin levels (AUC 0.8, Se 72.0, Sp 62, PPV 62, and NPV $72 \%$ ) [42]. Therefore, NAFIC score is promising but requires further validation.

3.5. Imaging Techniques. In a small study of 58 patients, magnetic resonance elastography (MRE) was highly accurate $($ AUC $=0.93)$ for discriminating patients with NASH from those with simple steatosis, with a sensitivity of $94 \%$ and a specificity of $73 \%$ [43]. Further validation is required, considering that MRE is costly and not widely available in routine clinical practice.

\section{Noninvasive Staging of Liver Fibrosis}

4.1. AST/ALT Ratio. The AST/ALT ratio is a simple noninvasive test that can detect cirrhosis with a reasonable accuracy as shown in patients with chronic hepatitis C [44]. Although data in patients with NAFLD are scarce, it is widely available and could be used in primary care. However, alcohol use should be excluded, as patients with alcoholic steatohepatitis would get a false positive result [45].

4.2. Ferritin. Ferritin is the primary tissue iron-storage in the liver but also an acute-phase protein; therefore it can be induced in both cases of iron overload and inflammatory systemic diseases [46].
Hyperferritinemia is prevalent in about $30 \%$ of subjects with NAFLD and can be the only altered laboratory parameter.

Whether high ferritin levels in patients with NAFLD are an expression of hepatic inflammation rather secondary to insulin resistance or represent true iron overload is still not clarified [47].

Indeed patients with NAFLD often have mild to moderate hepatic iron accumulation, and phlebotomies may improve insulin resistance [48-50].

Therefore, serum ferritin has also been proposed as a marker of both NASH and liver fibrosis.

In a study of 628 patients with NAFLD, serum ferritin levels greater than 1.5 times the upper value of normal were independent predictors of advanced fibrosis [51].

In another study of 482 patients with NAFLD, although hyperferritinemia was common, the extent of elevation did not correlate with the histological stage [52].

In a study of 1201 patients with biopsy-proven NAFLD, serum ferritin levels increased with increasing histological grade of steatosis, lobular inflammation, and ballooning; however its diagnostic performance for detecting presence of fibrosis was suboptimal (AUC of $0.6,0.57$, and 0.55 for detecting fibrosis, severe fibrosis, and advanced fibrosis, resp.) [53].

The above data suggest that serum ferritin cannot be used as a biomarker for NASH or fibrosis on its own; however it is incorporated in panels for liver fibrosis assessment as discussed later.

4.3. APRI. The aspartate aminotransferase to platelet ratio index (APRI) has been proposed as a simple test for staging fibrosis in several chronic liver diseases, firstly in $\mathrm{CHC}$ [54].

APRI has been designed to detect significant fibrosis (METAVIR $\geq \mathrm{F} 2$ ) and cirrhosis with dual cut-offs in both stages. Studies in patients with NAFLD are scarce compared to other diseases and have not always used the recommended cut-offs [55].

In a recent study involving 358 patients with biopsyproven NAFLD, the sensitivity and specificity of APRI with a cut-off $>1$ for significant fibrosis were, respectively, 30 and 93\% [56]. Therefore APRI, similar to other simple noninvasive panels, could be used to exclude significant fibrosis.

4.4. BARD. BARD score includes body mass index (BMI), AST/ALT ratio, and diabetes and has been designed to detect significant fibrosis (Brunt score $\geq$ F3) [57].

Its main limitation is its high false positivity, based on the overestimation of BMI and presence of diabetes.

Using a cut-off point $\geq 2$ the sensitivity for the detection of advanced fibrosis was between 86.8 and $100 \%$ while the specificity ranged from $32.5 \%$ to $34.7 \%$ in different studies [57-59].

Subsequently, an enhanced model of BARD was attempted by adding INR to the panel. This was tested in a small cohort of 107 patients and resulted in an improved composite score for sensitivity and specificity (AUC 0.88 versus 0.8) [59].

In a population of $242 \mathrm{NAFLD}$ patients, the BARD score had the lowest specificity, sensitivity, Youden index, and predictive values for predicting both significant fibrosis and 
cirrhosis when compared with other (simple and complex) models for diagnosis of liver fibrosis [60].

4.5. NAFLD Fibrosis Score. NAFLD fibrosis score was constructed and validated in a population of 733 patients with NAFLD for detecting significant fibrosis (Brunt score $\geq F 3$ ).

It consists of age, hyperglycemia, BMI, platelet count, albumin, and AST/ALT ratio and has dual cut-offs. The AUC for significant fibrosis is 0.88 and 0.82 , with sensitivity of $81 \%$ and $77 \%$ and specificity of 77 and $71 \%$ applying the low cutoff score, and sensitivity of 51 and $43 \%$ and specificity of 98 and $96 \%$ applying the high cut-off score in the estimation and validation group, respectively [61]. Therefore, this score can be used to triage patients in primary care, as it accurately diagnoses patients without significant fibrosis.

4.6. FIB-4. The FIB-4 index was developed as a noninvasive panel to detect significant fibrosis in subjects with HIVHCV coinfection and it has been independently validated in subjects with HCV infection [62, 63].

FIB-4 consists of age, AST, ALT, and platelet count. Similar to the NAFLD fibrosis score it has dual cut-offs, that is, a low cut-off of 1.45 with a high sensitivity and a high cutoff of 3.25 with a high specificity.

FIB-4 has showed a better accuracy in diagnosing advanced fibrosis in NAFLD patients when compared to other simple noninvasive tests in several studies (overall AUC $0.88)[64,65]$.

Both the NAFLD fibrosis score and FIB- 4 can be used in the primary care as triaging tests, in order to decide which patients do not have advanced fibrosis and therefore can be safely managed there.

4.7. Hepascore. Hepascore is a proprietary test that includes age, gender, bilirubin, gamma-glutamyl transferase (GGT), hyaluronic acid, and $\alpha 2$-macroglobulin [66] .

This scoring system has been tested in NAFLD population in a study of 242 patients. In comparison with other serum biomarkers and complex scores [60] it apparently had a good diagnostic accuracy for staging fibrosis (AUC, sensitivity, and specificity of $0.73,50.5 \%$, and $88.3 \%$ for stage $\geq F 2,0.81,75.5 \%$, and $84.1 \%$ for stage $\geq \mathrm{F} 3$, and $0.9,87 \%$, and $89 \%$ for cirrhosis, resp.). In another study it was showed that its reliability may vary depending on the influence of fasting on hyaluronic acid values [67].

4.8. ELF Test. The enhanced liver fibrosis (ELF) test is a simplified version of the original ELF (OELF) panel whose diagnostic performance has been validated for a variety of liver disorders [68].

ELF test is an algorithm that consists of hyaluronic acid (HA), amino-terminal propeptide of type III procollagen (PIIINP), and tissue inhibitor of metalloproteinase 1 (TIMP$1)$.

In the first validation study in a NAFLD/NASH population of 196 patients for the staging of liver fibrosis, the ELF panel had good performance in distinguishing severe fibrosis (stages 3-4) with an AUC of 0.9, but lower AUCs for moderate fibrosis and absence of fibrosis ( 0.82 and 0.76 , resp.)
[69]. Similar findings were confirmed in NAFLD pediatric populations [70,71].

4.9. FibroTest and FibroMeter. FibroTest is a biomarker of liver fibrosis initially validated in patients with chronic hepatitis C [72]. It consists of bilirubin, haptoglobin, GGT, $\alpha 2-$ macroglobulin, and apolipoprotein-A and classifies patients into 3 categories, namely, presence/absence of a certain stage of fibrosis or indeterminate values. Approximately a third of the patients have values in the indeterminate range [73].

The FibroMeter NAFLD score is a proprietary panel of serum markers that has shown a high diagnostic accuracy for the staging of fibrosis [74]. In a study of 235 NAFLD patients, the AUCs for the FibroMeter NAFLD score were 0.94 for significant fibrosis ( $\geq F 2), 0.93$ for severe fibrosis (F3), and 0.9 for cirrhosis, with high sensitivity, specificity, PPV, and NPV. It is easily calculated from simple parameters and employing optimal cut-off values for the diagnosis of significant fibrosis, $97.4 \%$ of patients would be correctly classified [75].

All the above proprietary panels need independent validation by groups not involved in their creation and also in non-Caucasian populations.

4.10. Simple Scores versus Complex Scores. In a multicenter study of 242 patients, complex models (Hepascore, FibroTest, and FIB-4) were compared to simple models (APRI, BARD). For the prediction of significant fibrosis $(\geq F 2)$ all these models had modest accuracy (AUC 0.71-0.74) with BARD being least accurate (AUC 0.61, $P<0.05$ versus others), while Hepascore and FIB- 4 had the highest specificity $(88.3 \%$ and $87.5 \%$, resp.). For the prediction of advanced fibrosis $(\geq F 3)$, all complex models provided specificity values $>80 \%$, with Hepascore providing the highest sensitivity (75.5\%), while all models (especially FIB-4 and Hepascore) had a good accuracy (AUC $0.8-0.86$ versus $0.7, P<0.05$ ).

For the prediction of cirrhosis, Hepascore provided the highest sensitivity (87\%). All models had reasonable specificity (>0.8) except for APRI. Complex scores had the best accuracy (AUC for Hepascore 0.94, FIB-4 and FibroTest 0.86) [60].

A recent meta-analysis has reported that FibroTest, ELF, and NAFLD fibrosis scores have significantly better diagnostic accuracy than BARD score and APRI, and their AUCs did not significantly differ from each other [6].

4.11. Imaging Techniques to Diagnose Fibrosis. Ultrasonography, CT scan, and MRI are commonly used in clinical practice for routine liver imaging. They can detect advanced cirrhosis with signs of portal hypertension (enlarged spleen and portal vein, and collateral venous circulation), but not fibrosis of lesser stages $[76,77]$.

Transient elastography (Fibroscan) is the most evaluated elastography-based technique for fibrosis assessment in NAFLD: it has been shown to be an accurate and reproducible methodology to discriminate patients without any degree of fibrosis from those with advanced fibrosis or cirrhosis both in adult and in pediatric NAFLD patients $[78,79]$.

A meta-analysis of 40 studies showed that elastography had good sensitivity and specificity for cirrhosis of different 
etiologies and less accuracy for lesser degrees of fibrosis but a precise validation of specific stiffness cut-off values for the various stages of fibrosis is still lacking [16].

Nevertheless this technique has some limitations. It can give falsely elevated values in certain conditions, such as acute hepatitis, extrahepatic cholestasis, hepatic congestion, congestive heart failure, hepatic amyloidosis, and recent food intake [80]. Moreover, using the conventional M probe, liver stiffness measurements are uninterpretable in $19 \%$ of the cases and this is mainly due to increased waist circumference and BMI, as it is frequently the case in patients with NAFLD [81].

A new dedicated XL probe for obese patients, which produces lower width shear waves, has been recently created. XL probe has similar overall diagnostic accuracy when compared to $\mathrm{M}$ probe with an AUC of $0.8-0.83$ for fibrosis $\geq F 2,0.85-0.87$ for $\geq F 3$, and $0.89-0.91$ for cirrhosis $[82,83]$. However, it generates lower stiffness values than the M probe; therefore different cut-offs should be used.

When using the $\mathrm{M}$ probe in all patients as first line and the XL probe in those failing the M probe measurements, 78-84\% of patients will have reliable liver stiffness measurements [84].

In a recent study, liver stiffness was measured by Fibroscan, ARFI, and SSI and the results were compared with histological data. Pairwise comparison of AUCs values between SSI, Fibroscan, and ARFI showed no significant difference in diagnosing mild fibrosis ( $\geq \mathrm{F} 1$ ) or cirrhosis (F4). SSI showed higher accuracy in diagnosing significant fibrosis $(\geq F 2)$ compared to ARFI and in diagnosing severe fibrosis $(\geq F 3)$ compared to Fibroscan [23].

Real-time (RT) elastography is another technique of interest, as shown in a cohort of 181 patients, with a diagnostic accuracy of 0.83-0.96 depending on the assessed fibrosis stage [85].

In a recent retrospective analysis of 142 patients, MRE showed good accuracy for the diagnosis of advanced fibrosis (AUC 0.95) with high sensitivity and specificity (0.85 and 0.93 , resp., at a cut-off of $4.15 \mathrm{kPa}$ ) [86]. These are probably the best results obtained by a noninvasive diagnostic technique for the present.

All elastography-based techniques need universally applicable validated cut-offs across different fibrosis stages. This would require adequately powered prospective studies.

\section{Genetic Markers as Potential Future Noninvasive Prognostic Tests}

It has also become increasingly apparent that genetic factors may influence the development and progression of NASH. These are polymorphisms of genes that regulate oxidative damage, the inflammatory cascade, and priming of fibrosis. The first such polymorphism described was a variant of patatin-like phospholipase-3 (PNPLA3) (also called adiponutrin), derived from an isoleucine-to-methionine substitution at residue 148 (single-nucleotide polymorphism or SNP). The GG genotype of PNPLA3 in patients with NAFLD has been associated with more severe steatosis, presence of NASH, and fibrosis $[87,88]$. Furthermore, it seems to correlate with an increased risk of atherogenesis and subsequent cardiovascular events [89]. Carriers of the PNPLA3 rs738409 minor (G) allele have also a higher probability to develop NAFLDcorrelated HCC, especially of the poorly differentiated type [90].

Another single nucleotide polymorphism which has been recently correlated with the development and prognosis of NAFLD is a glutamate to lysine amino acid substitution at residue 167 in transmembrane 6 superfamily member 2 (TM6SF2) sequence; the T allele of this gene is associated with the development of NAFLD, its progression to fibrosis and cirrhosis, and also altered cholesterol metabolism [91]; however there are some contradictions in literature and further studies are required to validate its role in larger cohorts of patients [92]. Further potential genetic determinants of NAFLD and progression of liver fibrosis are being evaluated in genome-wide association studies and may be used in the future as noninvasive prognostic indicators.

\section{Conclusions}

For the present several noninvasive diagnostic strategies have been proposed as alternatives to liver biopsy in patients with NAFLD, with various levels of diagnostic accuracy. Although the accurate diagnosis of NASH is still not possible with available noninvasive tools, there are several scores that can diagnose advanced fibrosis ( $\geq$ F3). Simple panels, such as FIB4 and NAFLD fibrosis score, are easily computable, have a high negative predictive value for advanced fibrosis, and have been validated against clinical outcomes. Therefore they could be used as first line test to "rule out" patients without advanced fibrosis and thus prevent unnecessary secondary care referrals in a significant number of patients.

More refined noninvasive tests such as Fibroscan, Hepascore, FibroMeter, FibroTest, or ELF could be used as second tier tests to further characterize patients, although this sequential approach needs prospective validation. The development and validation of such noninvasive algorithms will likely limit the need for liver biopsy in a selected few in the near future.

\section{Conflict of Interests}

The authors declare that there is no conflict of interests regarding the publication of this paper.

\section{References}

[1] E. A. Tsochatzis, S. Manolakopoulos, G. V. Papatheodoridis, and A. J. Archimandritis, "Insulin resistance and metabolic syndrome in chronic liver diseases: old entities with new implications," Scandinavian Journal of Gastroenterology, vol. 44, no. 1, pp. 6-14, 2009.

[2] H. Cortez-Pinto and M. E. Camilo, "Non-alcoholic fatty liver disease/non-alcoholic steatohepatitis (NAFLD/NASH): diagnosis and clinical course," Best Practice and Research: Clinical Gastroenterology, vol. 18, no. 6, pp. 1089-1104, 2004.

[3] N. Chalasani, Z. Younossi, J. E. Lavine et al., "The diagnosis and management of non-alcoholic fatty liver disease: practice 
guideline by the American Gastroenterological Association, American Association for the Study of Liver Diseases, and American College of Gastroenterology," Gastroenterology, vol. 142, no. 7, pp. 1592-1609, 2012.

[4] V. Ratziua, S. Bellentanib, H. Cortez-Pintoc, C. Dayd, and G. Marchesinie, "A position statement on NAFLD/NASH based on the EASL 2009 special conference," Journal of Hepatology, vol. 53, no. 2, pp. 372-384, 2010.

[5] R. Vuppalanchi and N. Chalasani, "Nonalcoholic fatty liver disease and nonalcoholic steatohepatitis: selected practical issues in their evaluation and management," Hepatology, vol. 49, no. 1, pp. 306-317, 2009.

[6] G. Musso, R. Gambino, M. Cassader, and G. Pagano, "Metaanalysis: natural history of non-alcoholic fatty liver disease (NAFLD) and diagnostic accuracy of non-invasive tests for liver disease severity," Annals of Medicine, vol. 43, no. 8, pp. 617-649, 2011.

[7] E. A. Tsochatzis, J. Bosch, and A. K. Burroughs, "Liver cirrhosis," The Lancet, vol. 383, no. 9930, pp. 1749-1761, 2014.

[8] E. Tsochatzis, G. V. Papatheodoridis, E. K. Manesis, G. Kafiri, D. G. Tiniakos, and A. J. Archimandritis, "Metabolic syndrome is associated with severe fibrosis in chronic viral hepatitis and non-alcoholic steatohepatitis," Alimentary Pharmacology and Therapeutics, vol. 27, no. 1, pp. 80-89, 2008.

[9] V. Papastergiou, E. Tsochatzis, and A. K. Burroughs, "Noninvasive assessment of liver fibrosis," Annals of Gastroenterology, vol. 25, no. 3, pp. 218-231, 2012.

[10] V. Ratziu, F. Charlotte, A. Heurtier et al., "Sampling variability of liver biopsy in nonalcoholic fatty liver disease," Gastroenterology, vol. 128, no. 7, pp. 1898-1906, 2005.

[11] L. B. Seeff, G. T. Everson, T. R. Morgan et al., "Complication rate of percutaneous liver biopsies among persons with advanced chronic liver disease in the HALT-C trial," Clinical Gastroenterology and Hepatology, vol. 8, no. 10, pp. 877-883, 2010.

[12] C. Crossan, E. A. Tsochatzis, L. Longworth et al., "Costeffectiveness of non-invasive methods for assessment and monitoring of liver fibrosis and cirrhosis in patients with chronic liver disease: systematic review and economic evaluation," Health Technology Assessment, vol. 19, no. 9, pp. 1-409, 2015.

[13] L. A. Adams, "Biomarkers of liver fibrosis," Journal of Gastroenterology and Hepatology, vol. 26, no. 5, pp. 802-809, 2011.

[14] S. M. Martínez, G. Crespo, M. Navasa, and X. Forns, "Noninvasive assessment of liver fibrosis," Hepatology, vol. 53, no. 1, pp. 325-335, 2011.

[15] Z. M. Younossi, M. Jarrar, C. Nugent et al., "A novel diagnostic biomarker panel for obesity-related nonalcoholic steatohepatitis (NASH)," Obesity Surgery, vol. 18, no. 11, pp. 1430-1437, 2008.

[16] E. A. Tsochatzis, K. S. Gurusamy, S. Ntaoula, E. Cholongitas, B. R. Davidson, and A. K. Burroughs, "Elastography for the diagnosis of severity of fibrosis in chronic liver disease: a metaanalysis of diagnostic accuracy," Journal of Hepatology, vol. 54, no. 4, pp. 650-659, 2011.

[17] European Association for the Study of the Liver, "EASL clinical practice guidelines: management of chronic hepatitis $B$ virus infection," Journal of Hepatology, vol. 57, no. 1, pp. 167-185, 2012.

[18] J. A. Carrión, M. Navasa, J. Bosch, M. Bruguera, R. Gilabert, and X. Forns, "Transient elastography for diagnosis of advanced fibrosis and portal hypertension in patients with hepatitis C recurrence after liver transplantation," Liver Transplantation, vol. 12, no. 12, pp. 1791-1798, 2006.
[19] F. Vizzutti, U. Arena, R. G. Romanelli et al., "Liver stiffness measurement predicts severe portal hypertension in patients with HCV-related cirrhosis," Hepatology, vol. 45, no. 5, pp. 12901297, 2007.

[20] L. Castéra, B. L. Bail, F. Roudot-Thoraval et al., "Early detection in routine clinical practice of cirrhosis and oesophageal varices in chronic hepatitis C: comparison of transient elastography (FibroScan) with standard laboratory tests and non-invasive scores," Journal of Hepatology, vol. 50, no. 1, pp. 59-68, 2009.

[21] R. Masuzaki, R. Tateishi, H. Yoshida et al., "Prospective risk assessment for hepatocellular carcinoma development in patients with chronic hepatitis C by transient elastography," Hepatology, vol. 49, no. 6, pp. 1954-1961, 2009.

[22] M. Yoneda, K. Suzuki, S. Kato et al., "Nonalcoholic fatty liver disease: US-based acoustic radiation force impulse elastography," Radiology, vol. 256, no. 2, pp. 640-647, 2010.

[23] C. Cassinotto, B. Lapuyade, A. Mouries et al., "Non-invasive assessment of liver fibrosis with impulse elastography: comparison of Supersonic Shear Imaging with ARFI and FibroScan," Journal of Hepatology, vol. 61, no. 3, pp. 550-557, 2014.

[24] M. Friedrich-Rust, D. Romen, J. Vermehren et al., "Acoustic radiation force impulse-imaging and transient elastography for non-invasive assessment of liver fibrosis and steatosis in NAFLD," European Journal of Radiology, vol. 81, no. 3, pp. e325e331, 2012.

[25] G. Ferraioli, C. Tinelli, M. Zicchetti et al., "Reproducibility of real-time shear wave elastography in the evaluation of liver elasticity," European Journal of Radiology, vol. 81, no. 11, pp. 3102-3106, 2012.

[26] S. K. Venkatesh, M. Yin, and R. L. Ehman, "Magnetic resonance elastography of liver: technique, analysis, and clinical applications," Journal of Magnetic Resonance Imaging, vol. 37, no. 3, pp. 544-555, 2013.

[27] M. Botros and K. A. Sikaris, "The de ritis ratio: the test of time," The Clinical Biochemist Reviews, vol. 34, no. 3, pp. 117-130, 2013.

[28] P. Mofrad, M. J. Contos, M. Haque et al., "Clinical and histologic spectrum of nonalcoholic fatty liver disease associated with normal ALT values," Hepatology, vol. 37, no. 6, pp. 1286-1292, 2003.

[29] S. Verma, D. Jensen, J. Hart, and S. R. Mohanty, "Predictive value of ALT levels for non-alcoholic steatohepatitis (NASH) and advanced fibrosis in non-alcoholic fatty liver disease (NAFLD)," Liver International, vol. 33, no. 9, pp. 1398-1405, 2013.

[30] D. M. Torres and S. A. Harrison, "NAFLD: predictive value of ALT levels for NASH and advanced fibrosis," Nature Reviews Gastroenterology and Hepatolog, vol. 10, no. 9, pp. 510-511, 2013.

[31] S. S. Kunde, A. J. Lazenby, R. H. Clements, and G. A. Abrams, "Spectrum of NAFLD and diagnostic implications of the proposed new normal range for serum ALT in obese women," Hepatology, vol. 42, no. 3, pp. 650-656, 2005.

[32] A. E. Feldstein and G. J. Gores, "Apoptosis in alcoholic and nonalcoholic steatohepatitis," Frontiers in Bioscience, vol. 10, no. 3, pp. 3093-3099, 2005.

[33] A. E. Feldstein, A. Wieckowska, A. R. Lopez, Y.-C. Liu, N. N. Zein, and A. J. McCullough, "Cytokeratin-18 fragment levels as noninvasive biomarkers for nonalcoholic steatohepatitis: a multicenter validation study," Hepatology, vol. 50, no. 4, pp. 1072-1078, 2009.

[34] A. E. Feldstein, N. Alkhouri, R. de Vito, A. Alisi, R. Lopez, and V. Nobili, "Serum cytokeratin-18 fragment levels are useful biomarkers for nonalcoholic steatohepatitis in children," The 
American Journal of Gastroenterology, vol. 108, no. 9, pp. 15261531, 2013.

[35] K. Cusi, Z. Chang, S. Harrison et al., "Limited value of plasma cytokeratin-18 as a biomarker for NASH and fibrosis in patients with non-alcoholic fatty liver disease," Journal of Hepatology, vol. 60, no. 1, pp. 167-174, 2014.

[36] S. Tanwar, P. M. Trembling, I. N. Guha et al., "Validation of terminal peptide of procollagen III for the detection and assessment of nonalcoholic steatohepatitis in patients with nonalcoholic fatty liver disease," Hepatology, vol. 57, no. 1, pp. 103-111, 2013.

[37] Z. M. Younossi, S. Page, N. Rafiq et al., "A biomarker panel for non-alcoholic steatohepatitis (NASH) and NASH-Related fibrosis," Obesity Surgery, vol. 21, no. 4, pp. 431-439, 2011.

[38] T. Poynard, V. Ratziu, F. Charlotte et al., "Diagnostic value of biochemical markers (Nash Test) for the prediction of non alcoholo steato hepatitis in patients with non-alcoholic fatty liver disease," BMC Gastroenterology, vol. 6, article 34, 2006.

[39] V. Ratziu, P. Giral, M. Munteanu et al., "Screening for liver disease using non-invasive biomarkers (FibroTest, SteatoTest and NashTest) in patients with hyperlipidaemia," Alimentary Pharmacology and Therapeutics, vol. 25, no. 2, pp. 207-218, 2007.

[40] G. Lassailly, R. Caiazzo, A. Hollebecque et al., "Validation of noninvasive biomarkers (FibroTest, SteatoTest, and NashTest) for prediction of liver injury in patients with morbid obesity," European Journal of Gastroenterology and Hepatology, vol. 23, no. 6, pp. 499-506, 2011.

[41] Y. Sumida, M. Yoneda, H. Hyogo et al., "A simple clinical scoring system using ferritin, fasting insulin, and type IV collagen $7 \mathrm{~S}$ for predicting steatohepatitis in nonalcoholic fatty liver disease," Journal of Gastroenterology, vol. 46, no. 2, pp. 257-268, 2011.

[42] A. Nakamura, M. Yoneda, Y. Sumida et al., "Modification of a simple clinical scoring system as a diagnostic screening tool for non-alcoholic steatohepatitis in Japanese patients with nonalcoholic fatty liver disease," Journal of Diabetes Investigation, vol. 4, no. 6, pp. 651-658, 2013.

[43] J. Chen, J. A. Talwalkar, M. Yin, K. J. Glaser, S. O. Sanderson, and R. L. Ehman, "Early detection of nonalcoholic steatohepatitis in patients with nonalcoholic fatty liver disease by using MR elastography," Radiology, vol. 259, no. 3, pp. 749-756, 2011.

[44] S. G. Sheth, S. L. Flamm, F. D. Gordon, and S. Chopra, "AST/ALT ratio predicts cirrhosis in patients with chronic hepatitis C virus infection," American Journal of Gastroenterology, vol. 93, no. 1, pp. 44-48, 1998.

[45] D. Sorbi, J. Boynton, and K. D. Lindor, "The ratio of aspartate aminotransferase to alanine aminotransferase: potential value in differentiating nonalcoholic steatohepatitis from alcoholic liver disease," American Journal of Gastroenterology, vol. 94, no. 4, pp. 1018-1022, 1999.

[46] E. Corradini and A. Pietrangelo, "Iron and steatohepatitis," Journal of Gastroenterology and Hepatology, vol. 27, supplement 2, pp. 42-46, 2012.

[47] P. Trombini and A. Piperno, "Ferritin, metabolic syndrome and NAFLD: elective attractions and dangerous liaisons," Journal of Hepatology, vol. 46, no. 4, pp. 549-552, 2007.

[48] L. Valenti, A. L. Fracanzani, E. Bugianesi et al., "HFE Genotype, Parenchymal Iron Accumulation, and Liver Fibrosis in Patients With Nonalcoholic Fatty Liver Disease," Gastroenterology, vol. 138, no. 3, pp. 905-912, 2010.

[49] J. E. Nelson, L. Wilson, E. M. Brunt et al., "Relationship between the pattern of hepatic iron deposition and histological severity in nonalcoholic fatty liver disease," Hepatology, vol. 53, no. 2, pp. 448-457, 2011.

[50] B. D. Maliken, J. E. Nelson, H. M. Klintworth, M. Beauchamp, M. M. Yeh, and K. V. Kowdley, "Hepatic reticuloendothelial system cell iron deposition is associated with increased apoptosis in nonalcoholic fatty liver disease," Hepatology, vol. 57, no. 5, pp. 1806-1813, 2013.

[51] K. V. Kowdley, P. Belt, L. A. Wilson et al., "Serum ferritin is an independent predictor of histologic severity and advanced fibrosis in patients with nonalcoholic fatty liver disease," Hepatology, vol. 55, no. 1, pp. 77-85, 2012.

[52] N. Chandok, G. Minuk, M. Wengiel, and J. Uhanova, "Serum ferritin levels do not predict the stage of underlying nonalcoholic fatty liver disease," Journal of Gastrointestinal and Liver Diseases, vol. 21, no. 1, pp. 53-58, 2012.

[53] M. Yoneda, E. Thomas, Y. Sumida et al., "Clinical usage of serum ferritin to assess liver fibrosis in patients with non-alcoholic fatty liver disease: proceed with caution," Hepatology Research, vol. 44, no. 14, pp. E499-E502, 2014.

[54] C.-T. Wai, J. K. Greenson, R. J. Fontana et al., "A simple noninvasive index can predict both significant fibrosis and cirrhosis in patients with chronic hepatitis C," Hepatology, vol. 38, no. 2, pp. 518-526, 2003.

[55] Y. Yilmaz, O. Yonal, R. Kurt, M. Bayrak, B. Aktas, and O. Ozdogan, "Noninvasive assessment of liver fibrosis with the aspartate transaminase to platelet ratio index (APRI): Usefulness in patients with chronic liver disease: APRI in chronic liver disease," Hepatitis Monthly, vol. 11, no. 2, pp. 103-107, 2011.

[56] E. B. Tapper, K. Krajewski, M. Lai et al., "Simple non-invasive biomarkers of advanced fibrosis in the evaluation of nonalcoholic fatty liver disease," Gastroenterology Report, vol. 2, no. 4, pp. 276-280, 2014.

[57] S. A. Harrison, D. Oliver, H. L. Arnold, S. Gogia, and B. A. Neuschwander-Tetri, "Development and validation of a simple NAFLD clinical scoring system for identifying patients without advanced disease," Gut, vol. 57, no. 10, pp. 1441-1447, 2008.

[58] M. Yoneda, K. Fujita, M. Inamori, A. Nakajima, M. Tamano, and H. Hiraishi, "Transient elastography in patients with nonalcoholic fatty liver disease (NAFLD)," Gut, vol. 56, no. 9, pp. 1330-1331, 2007.

[59] T. H. Lee, S. H. Han, J. D. Yang, D. Kim, and M. Ahmed, "Prediction of advanced fibrosis in nonalcoholic fatty liver disease: an enhanced model of BARD score," Gut and Liver, vol. 7, no. 3, pp. 323-328, 2013.

[60] L. A. Adams, J. George, E. Bugianesi et al., "Complex noninvasive fibrosis models are more accurate than simple models in non-alcoholic fatty liver disease," Journal of Gastroenterology and Hepatology, vol. 26, no. 10, pp. 1536-1543, 2011.

[61] P. Angulo, J. M. Hui, G. Marchesini et al., "The NAFLD fibrosis score: a noninvasive system that identifies liver fibrosis in patients with NAFLD," Hepatology, vol. 45, no. 4, pp. 846-854, 2007.

[62] R. K. Sterling, E. Lissen, N. Clumeck et al., "Development of a simple noninvasive index to predict significant fibrosis in patients with HIV/HCV coinfection," Hepatology, vol. 43, no. 6, pp. 1317-1325, 2006.

[63] A. Vallet-Pichard, V. Mallet, B. Nalpas et al., "FIB-4: an inexpensive and accurate marker of fibrosis in HCV infection. Comparison with liver biopsy and FibroTest," Hepatology, vol. 46, no. 1, pp. 32-36, 2007. 
[64] Y. Sumida, M. Yoneda, H. Hyogo et al., "Validation of the FIB4 index in a Japanese nonalcoholic fatty liver disease population," BMC Gastroenterology, vol. 12, article 2, 2012.

[65] A. G. Shah, A. Lydecker, K. Murray, B. N. Tetri, M. J. Contos, and A. J. Sanyal, "Comparison of noninvasive markers of fibrosis in patients with nonalcoholic fatty liver disease," Clinical Gastroenterology and Hepatology, vol. 7, no. 10, pp. 1104-1112, 2009.

[66] J. Guéchot, E. Lasnier, N. Sturm, A. Paris, and J.-P. Zarski, "Automation of the Hepascore and validation as a biochemical index of liver fibrosis in patients with chronic hepatitis $\mathrm{C}$ from the ANRS HC EP 23 Fibrostar cohort," Clinica Chimica Acta, vol. 411, no. 1-2, pp. 86-91, 2010.

[67] E. Rossi, L. A. Adams, H. L. Ching, M. Bulsara, G. C. MacQuillan, and G. P. Jeffrey, "High biological variation of serum hyaluronic acid and hepascore, a biochemical marker model for the prediction of liver fibrosis," Clinical Chemistry and Laboratory Medicine, vol. 51, no. 5, pp. 1107-1114, 2013.

[68] W. M. C. Rosenberg, M. Voelker, R. Thiel et al., "Serum markers detect the presence of liver fibrosis: a cohort study," Gastroenterology, vol. 127, no. 6, pp. 1704-1713, 2004.

[69] I. N. Guha, J. Parkes, P. Roderick et al., "Noninvasive markers of fibrosis in nonalcoholic fatty liver disease: validating the European liver fibrosis panel and exploring simple markers," Hepatology, vol. 47, no. 2, pp. 455-460, 2008.

[70] J. Parkes, I. N. Guha, P. Roderick et al., "Enhanced Liver Fibrosis (ELF) test accurately identifies liver fibrosis in patients with chronic hepatitis C," Journal of Viral Hepatitis, vol. 18, no. 1, pp. 23-31, 2011.

[71] V. Nobili, J. Parkes, G. Bottazzo et al., "Performance of ELF serum markers in predicting fibrosis stage in pediatric nonalcoholic fatty liver disease," Gastroenterology, vol. 136, no. 1, pp. 160-167, 2009.

[72] T. Poynard, R. Morra, P. Halfon et al., "Meta-analyses of FibroTest diagnostic value in chronic liver disease," $B M C$ Gastroenterology, vol. 7, article 40, 2007.

[73] V. Ratziu, J. Massard, F. Charlotte et al., "Diagnostic value of biochemical markers (Fibro Test-FibroSURE) for the prediction of liver fibrosis in patients with non-alcoholic fatty liver disease," BMC Gastroenterology, vol. 6, article 6, 2006.

[74] D. Festi, R. Schiumerini, L. Marzi et al., "Review article: the diagnosis of non-alcoholic fatty liver disease-availability and accuracy of non-invasive methods," Alimentary Pharmacology and Therapeutics, vol. 37, no. 4, pp. 392-400, 2013.

[75] P. Calès, F. Lainé, J. Boursier et al., "Comparison of blood tests for liver fibrosis specific or not to NAFLD," Journal of Hepatology, vol. 50, no. 1, pp. 165-173, 2009.

[76] J. K. Dyson, S. McPherson, and Q. M. Anstee, "Non-alcoholic fatty liver disease: non-invasive investigation and risk stratification," Journal of Clinical Pathology, vol. 66, no. 12, pp. 1033-1045, 2013.

[77] G. Ligabue, G. Besutti, R. Scaglioni et al., "MR quantitative biomarkers of non-alcoholic fatty liver disease: technical evolutions and future trends," Quantitative Imaging in Medicine and Surgery, vol. 3, no. 4, pp. 192-195, 2013.

[78] V. W.-S. Wong, J. Vergniol, G. L.-H. Wong et al., "Diagnosis of fibrosis and cirrhosis using liver stiffness measurement in nonalcoholic fatty liver disease," Hepatology, vol. 51, no. 2, pp. 454-462, 2010.

[79] V. Nobili, F. Vizzutti, U. Arena et al., "Accuracy and reproducibility of transient elastography for the diagnosis of fibrosis in pediatric nonalcoholic steatohepatitis," Hepatology, vol. 48, no. 2, pp. 442-448, 2008.

[80] G. L. Wong, "Update of liver fibrosis and steatosis with transient elastography (Fibroscan)," Gastroenterology Report, vol. 1, no. 1, pp. 19-26, 2013.

[81] L. Castéra, J. Foucher, P.-H. Bernard et al., "Pitfalls of liver stiffness measurement: a 5-year prospective study of 13,369 examinations," Hepatology, vol. 51, no. 3, pp. 828-835, 2010.

[82] V. W.-S. Wong, J. Vergniol, G. L.-H. Wong et al., "Liver stiffness measurement using XL probe in patients with nonalcoholic fatty liver disease," American Journal of Gastroenterology, vol. 107, no. 12, pp. 1862-1871, 2012.

[83] R. Sirli, I. Sporea, A. Deleanu et al., "Comparison between the $\mathrm{M}$ and XL probes for liver fibrosis assessment by transient elastography," Medical Ultrasonography, vol. 16, no. 2, pp. 119122, 2014.

[84] W. Kemp and S. Roberts, "Feasibility and performance of the FibroScan XL probe," Hepatology, vol. 55, no. 4, pp. 1308-1309, 2012.

[85] H. Ochi, M. Hirooka, Y. Koizumi et al., "Real-time tissue elastography for evaluation of hepatic fibrosis and portal hypertension in nonalcoholic fatty liver diseases," Hepatology, vol. 56, no. 4, pp. 1271-1278, 2012.

[86] D. Kim, W. R. Kim, J. A. Talwalkar, H. J. Kim, and R. L. Ehman, "Advanced fibrosis in nonalcoholic fatty liver disease: noninvasive assessment with MR elastography," Radiology, vol. 268, no. 2, pp. 411-419, 2013.

[87] L. Valenti, A. Al-Serri, A. K. Daly et al., "Homozygosity for the patatin-like phospholipase-3/adiponutrin I148M polymorphism influences liver fibrosis in patients with nonalcoholic fatty liver disease," Hepatology, vol. 51, no. 4, pp. 1209-1217, 2010.

[88] Y. Rotman, C. Koh, J. M. Zmuda, D. E. Kleiner, and T. J. Liang, "The association of genetic variability in patatin-like phospholipase domain-containing protein 3 (PNPLA3) with histological severity of nonalcoholic fatty liver disease," Hepatology, vol. 52, no. 3, pp. 894-903, 2010.

[89] S. Petta, L. Valenti, G. Marchesini et al., "PNPLA3 GG genotype and carotid atherosclerosis in patients with non-alcoholic fatty liver disease," PLoS ONE, vol. 8, no. 9, Article ID e74089, 2013.

[90] Y.-L. Liu, G. L. Patman, J. B. S. Leathart et al., "Carriage of the PNPLA3 rs738409 $\mathrm{C}>\mathrm{G}$ polymorphism confers an increased risk of non-alcoholic fatty liver disease associated hepatocellular carcinoma," Journal of Hepatology, vol. 61, no. 1, pp. 75-81, 2014.

[91] Y. L. Liu, H. L. Reeves, A. D. Burt et al., “TM6SF2 rs58542926 influences hepatic fibrosis progression in patients with nonalcoholic fatty liver disease," Nature Communications, vol. 5, article 4309, 2014.

[92] S. Sookoian, G. O. Castaño, R. Scian et al., "Genetic variation in transmembrane 6 superfamily member 2 and the risk of nonalcoholic fatty liver disease and histological disease severity," Hepatology, vol. 61, no. 2, pp. 515-525, 2015. 


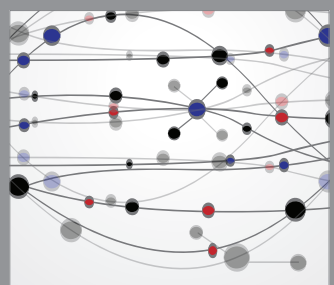

The Scientific World Journal
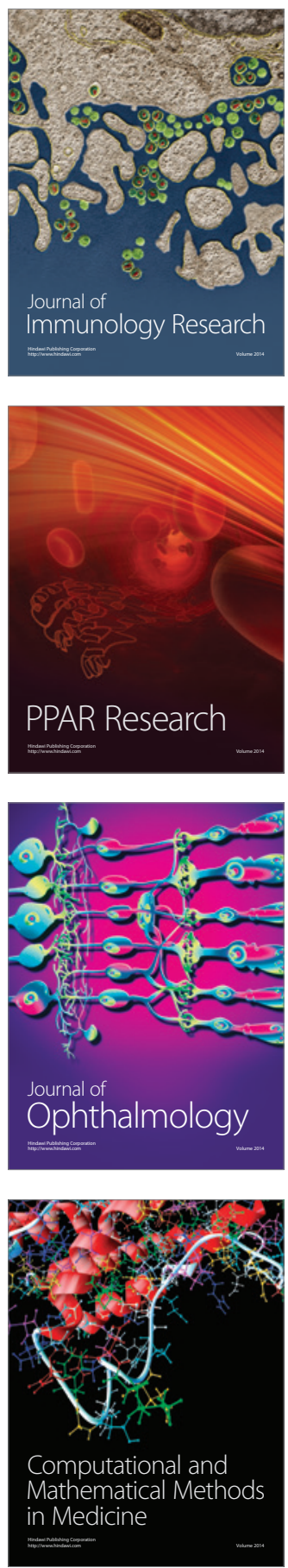

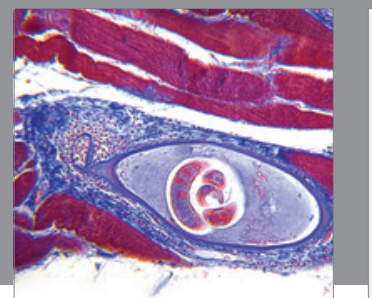

Gastroenterology

Research and Practice
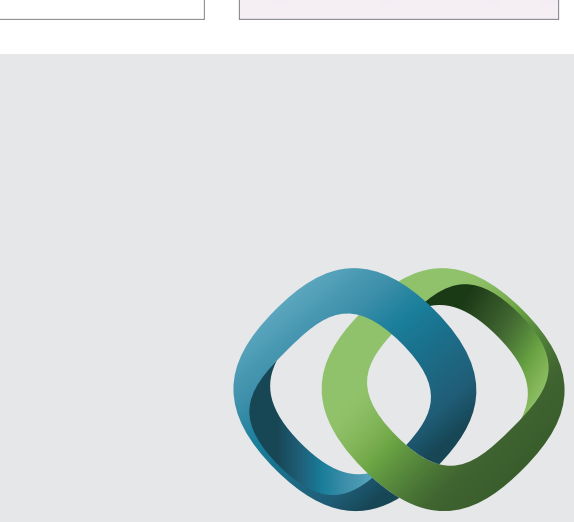

\section{Hindawi}

Submit your manuscripts at

http://www.hindawi.com
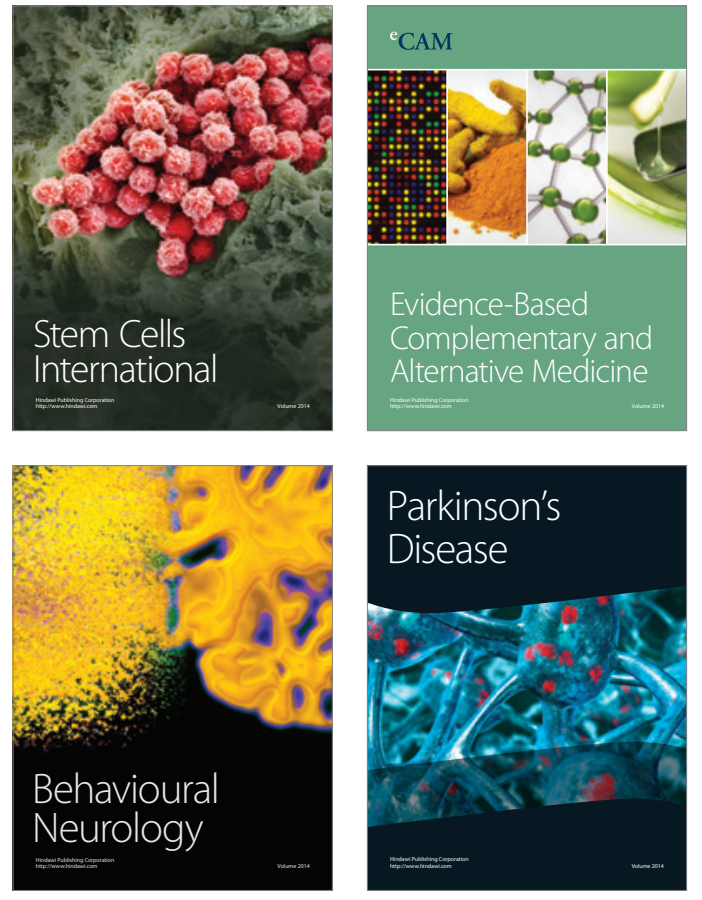
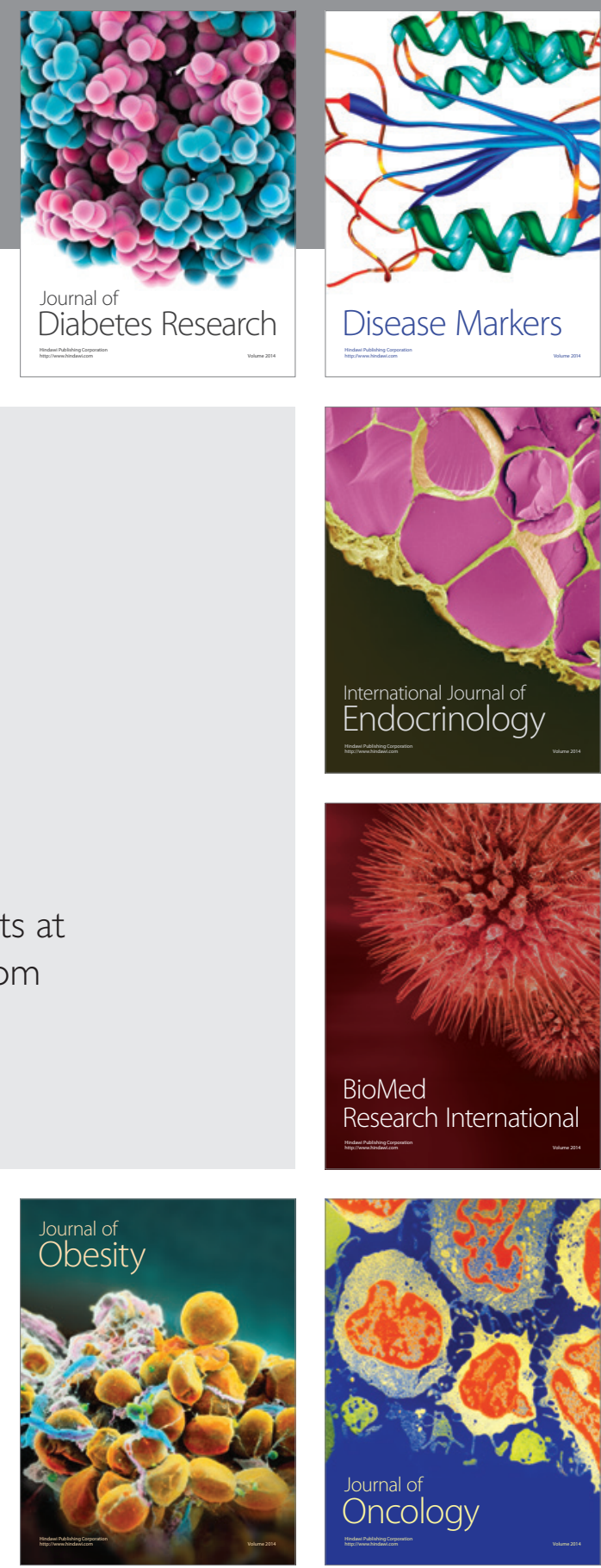

Disease Markers
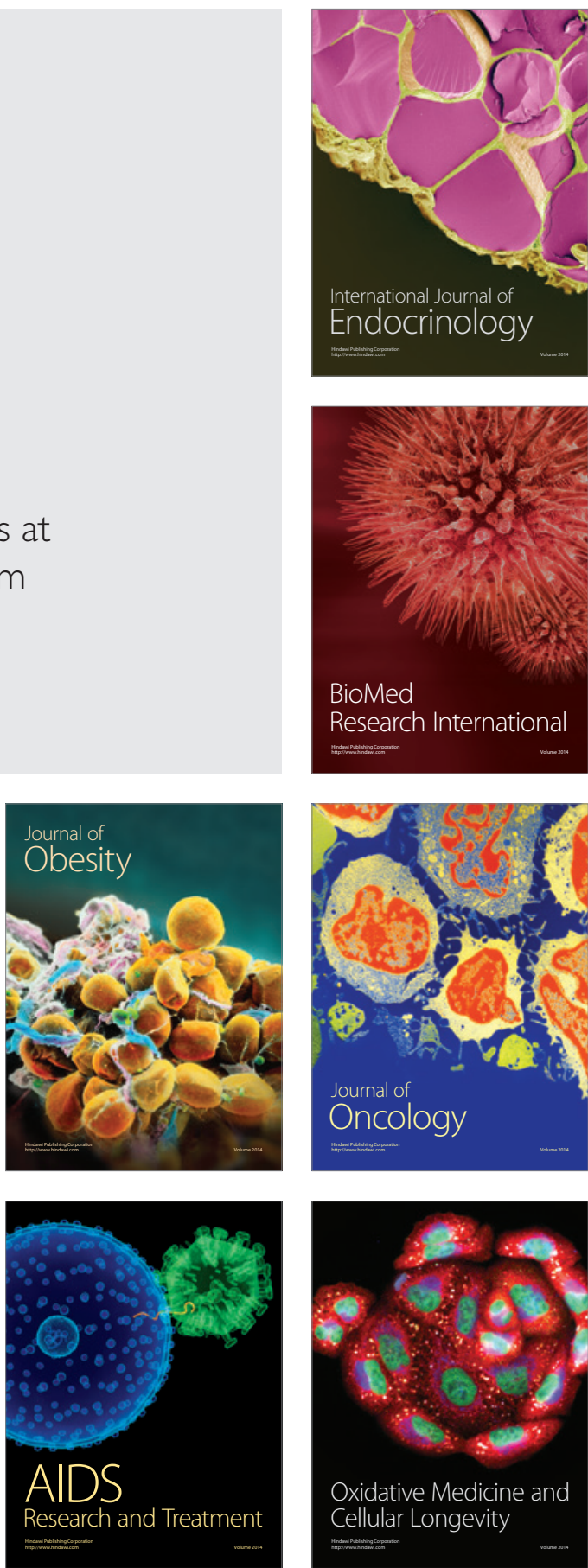